\title{
Efectos de la aplicación de subsidios al mercado porcino en México
}

\author{
Rebollar, R.S. ${ }^{1}$; Velázquez, V.H.H. ${ }^{1,2}$; Gómez, T.G. ${ }^{1,3}$;Posadas, D.R.R. ${ }^{2}$ y Martínez, C.F.E. ${ }^{3}$
}

Universidad Autónoma del Estado de México. Centro Universitario UAEM Temascaltepec. México.

2Universidad Autónoma de Hidalgo. Campus Zimapan. Hidalgo. México.

3Universidad Autónoma del Estado de México. Instituto de Investigación en Ciencias Agropecuarias y Rurales. Toluca. Estado de México. México.

Palabras ClaVE AdicIONALES

Carne de cerdo.

Canal.

Programación no lineal.

Valor Social Neto.

\section{RESUMEN}

El objetivo de esta investigación fue evaluar el efecto de la aplicación de subsidios sobre el mercado de la carne de porcino en canal en México 2015 y 2016, a nivel región, mediante un modelo de programación no lineal. Se utilizaron ocho regiones productoras, dos puntos de internación de importaciones y ocho regiones consumidoras: Noroeste, Norte, Noreste, Centro-oeste, Centroeste, Sur, Oriente, Península, Punto de internación uno, Punto de internación dos; el análisis se realizó en producción, importaciones y consumo. Bajo condiciones óptimas el modelo subestimó producción nacional en $0,4 \%$, sobreestimó importaciones y consumo regional-nacional en 2,5 y $0,4 \%$ con un Valor Social Neto (VSN) óptimo de 216,563 millones de dólares. El subsidio a la oferta de carne de cerdo incrementaría la producción y consumo nacional e importaciones en 0,07, 0,006 y $0.03 \%$, el VSN aumentaría en $0,006 \%$. A nivel región, los precios óptimos al productor y al consumidor se reducirían debido al efecto del subsidio. Se concluye que, un subsidio a la función de oferta de carne de cerdo en canal, mejoraría el beneficio de la sociedad, incrementando la producción y protegiendo a consumidores. La políitca podría ser viable en favor de la producción, siempre y cuando, el objetivo gubernamental se encamine hacia ese sector.

\section{Effects to the application of subsidies to the pork market in Mexico}

\section{SUMMARY}

\section{ADDITIONAL KEYWORDS}

Pork meat.

Carcass.

Nonlinear programming.

Social Net Value.

\section{INFORMATION}

Cronología del artículo.

Recibido/Received: 26.10.2017

Aceptado/Accepted: 02.12.2019

On-line: 15.01.2020

Correspondencia a los autores/Contact e-mail:

samrere@hotmail.com

\section{INTRODUCCIÓN}

Uno de los principios fundamentales en la economía, es que los subsidios representan apoyos monetarios o en especie que otorgan los gobiernos a la producción o al consumo (Parkin y Loria 2015). Los subsidios se otorgan a bienes considerados de primera necesidad, como agua, energía, alimentación, bienes agropecuarios, o a bienes que son preferentes como educación, servicios médicos, entre algunos otros.

Según Graue (2006), un bien o servicio preferente es aquel cuyo consumo se considera deseable por el gobierno, debido a que genera bienestar no sólo al consumidor sino a toda la sociedad; por tanto, se fomenta su consumo a través de regulaciones y subsidios. El 
subsidio, se le entrega al productor para que a su vez, lo traslade al consumidor para que incremente su competitividad o para que mejore su calidad de vida, dicho apoyo puede beneficiar en mayor o menor medida tanto al productor como al consumidor, en función a la elasticidad de la demanda y de la oferta (ceterisparibus).

Cuando se trata de un bien con elasticidad unitaria en ambas variables (en la demanda y en la oferta), la mitad del subsidio se le otorga al consumidor y la otra mitad a la producción o al productor; cuando la demanda es inelástica, es el consumidor quien recibe la mayor parte del subsidio y cuando es perfectamente inelástica, el subsidio recae totalmente en el consumidor, tal es el caso de bienes de primera necesidad y preferentes sin sustitutos (Graue 2006; Parkin y Loría 2015).

Los subsidios no pueden catalogarse en el ámbito de buenos o malos, sino como ventajosos o perjudiciales, según cómo cumplan los objetivos para los que se crearon; con los subsidios, los consumidores ganan al obtener productos necesarios a precios bajos; el productor también gana, porque el precio de los bienes y la cantidad vendida son mayores; sin embargo, debe entenderse que no todo el tiempo es así; todo dependerá de la manera en que se realice el análisis del subsidio; es decir, si el monto del apoyo se adiciona a los costos de transporte o bien, se elige adicionarlo a la función de oferta del productor; tal es el caso de la porcicultura mexicana, en donde cada año, las empresas engordadoras que entregan a la agroindustria cerdos finalizados con un peso de $120 \mathrm{~kg}$, reciben hasta un peso más por kilogramo $(\mathrm{kg})$ como incentivo por peso óptimo del animal(FIRA 2017).

Trabajos de investigación que discuten el efecto de subsidios en la porcicultura, son casi nulos; sin embargo, Velázquez et al. (2016), utilizaron un modelo de equilibrio parcial sobre el mercado porcino en México, en el que demostraron que cuando el monto del subsidio se adiciona a costos de transporte, tanto productores como consumidores se benefician, el bienestar de la sociedad se incrementa con relación al modelo óptimo, pero el análisis bajo esa forma, se considera sólo de corto plazo.

Al respecto, la carne de cerdo es de las más producidas a nivel mundial, en 2013 tal volumen fue 108,4 millones de toneladas (Mt) y en abril de 2017 cercano a 110,7 Mt, es decir, un incremento promedio de 0,5\% (PORCIMEX 2017). En 2016, el principal productor mundial de carne de cerdo fue China, con 52,9Mt (48,2\%), la Unión Europea (UE) con 23,4 Mt (21,3\%) y, en tercer lugar, Estados Unidos con 11,3 Mt (10,3\%). En consumo, la carne de cerdo, se reporta como la más demandada en el mundo, la medición de esta variable se realiza a través de la disponibilidad per cápita, en la que Hong Kong y China, sobresalen en el mundo con 67,7 y 42,5 kilogramos (kg) por persona por año, aunque los más productores del orbe, también son los mayores consumidores (China, UE y Estados Unidos) (PORCIMEX 2017).

En México, la producción de carne de cerdo la realizan 5800 productores y, ha ocupado siempre una posición importante dentro las carnes de especies pecua- rias de interés económico; reportes oficiales señalaron incrementos en su volumen de $0,5 \%$ entre 2013 y 2014 , crecimiento inferior al del PIB, al pasar de un millón 200 mil toneladas (t) y 2,4\% de 2014 a 2015 al pasar de un millón 290 mil t a un millón 320 mil. Hasta abril de 2017, México colaboró con poco más de 1,4 Mt (1,42 Mt) al total mundial, posicionándose en el lugar décimo sexto. Por entidad federativa, en este mismo periodo, Jalisco, Sonora, Puebla, Yucatán, Veracruz, Guanajuato y Michoacán han aportado alrededor de 898,9 mil t (65,9\%) (SENASICA 2015; PORCIMEX 2017).

En 2016, el consumo nacional aparente (CNA) fue 2,1 Mt, equivalente a una disponibilidad per cápita de 16,2 kg (CONAPO 2016; SNIIM 2016; PORCIMEX 2017); los principales consumidores fueron Ciudad de México (CDMX) y Estado de México.

Por lo anterior, el objetivo de este trabajo fue evaluar el efecto de la aplicación de subsidios, sobre producción, consumo e importaciones de carne de cerdo en canal en México de forma regional; cuantificando tal efecto sobre la medida de bienestar, conocida como Valor Social Neto (VSN). La hipótesis central, supone que ante ofertas y demandas regionales inelásticas, cuando el subsidio se adiciona dentro de la función de oferta en cada una de las regiones, el nivel de bienestar social se incrementa; el precio al productor y al consumidor disminuye, la cantidad ofrecida aumenta, en tanto que el volumen de importaciones y el consumo de esta carne, así como el bienestar de la sociedad se incrementan con relación al modelo base.

\section{MATERIAL Y MÉTODOS}

Se utilizó un modelo de equilibrio parcial, con programación no lineal (programación cuadrática), sin almacenamiento; cuya función objetivo (función de Valor Social Neto) consistió en maximizar el área bajo las curvas de demanda, menos el área bajo las curvas de oferta, menos los costos de comercialización, de la carne de cerdo en canal nacional como de la carne importada de esta misma especie pecuaria, sujeta a un conjunto de restricciones de oferta y demanda. El modelo se aplicó al mercado nacional de la carne de cerdo en canal, dividido en regiones, con datos observados y obtenidos en 2015.

Para ello, con base en Bassols (1999) y Rebollar et al. (2014), el país se dividió en ocho regiones productoras, ocho regiones consumidoras (Tabla I) y dos puntos de internación de las importaciones, mismos que se agregaron a la matriz de regiones productoras, para dar un total de 10.

El punto de internación uno (PI1), se integró por las aduanas de Colombia en el estado de Nuevo León, Nuevo Laredo y Reynosa por parte del estado de Tamaulipas y Piedras Negras en el estado de Coahuila. La fuente oficial señaló que este primer punto de internación, registra el ingreso del 90,1\% de la carne importada de carne de cerdo que llega a los centros consumidores de México. El punto de internación dos (PI2), se integró por las aduanas de Mexicali y Tijuana en el estado de Baja California; Nogales y San Luis Río Colorado pertenecientes al estado de Sonora y Ciudad 


\begin{tabular}{ll}
\hline \multicolumn{2}{l}{ Tabla I. Regiones y entidades de México utilizadas en el análisis (Regions and entities of Mexico used in the analysis). } \\
\hline Región & Estados \\
\hline Noroeste (NO) & Baja California, Baja California Sur, Sonora, Sinaloa y Nayarit \\
Norte (NR) & Chihuahua, Coahuila, Durango, San Luis Potosí, Zacatecas \\
Noreste (NE) & Nuevo León y Tamaulipas \\
Centro-occidente (CO) & Aguascalientes, Colima, Guanajuato, Jalisco, Michoacán \\
Centro-este (CE) & Ciudad de México, Hidalgo, Estado de México, Morelos, Puebla, Querétaro, Tlaxcala \\
Sur (SU) & Chiapas, Oaxaca y Guerrero \\
Oriente (OR) & Veracruz y Tabasco \\
Península de Yucatán (PE) & Campeche, Yucatán y Quintana Roo \\
\hline
\end{tabular}

Bassols (1999:43); Rebollar et al. (2014).

Juárez en el estado de Chihuahua; por este segundo punto de internación, ingresa el 9,9\% de la importación de carne de cerdo (SIAP 2016). En este trabajo, se consideró que todas las importaciones de esta carne, provinieron de Estados Unidos de Norteamérica. Así, se conformaron 10 regiones productoras, ocho del interior de México y dos constituidas por los puntos de internación ya mencionados.

En el modelo de equilibrio espacial de precios, se utilizaron ofertas y demandas inelásticas (Rebollar et al. 2014). Los modelos son, funcionalmente, dependientes del precio, que en la literatura se conocen como funciones inversas de demanda y funciones inversas de oferta (Martínez y Hernández 2012).

Para la determinación del VSN, fue necesario la definición endógena de precios, por lo que se empleó la función inversa de la demanda (Martínez y Hernández 2012), que para la región $i$ fue:

$$
P_{d i}=P_{d i}\left(Y_{d i}\right)=\lambda_{d i}+\omega_{d i} Y_{d i} ; \omega<0 ；
$$

Donde:

$P_{d i}=$ precio de demanda de carne de cerdo en canal, en la región $i$, en pesos por tonelada

$Y_{d i}=$ cantidad demandada de carne de cerdo en canal en la región $i$, en toneladas

$\lambda=$ intercepto de la función de demanda de carne de cerdo en canal para la región $i$

$\omega=$ pendiente de la función de demanda de carne de cerdo en canal para la región $i$.

Para la misma región y en referencia a la oferta, el subsidio se insertó en la función de oferta dependiente del precio (Graue 2006), de la siguiente forma:

$$
X_{s}^{\prime}=\theta_{s}+\gamma_{s}\left(P_{s}+S u b\right)
$$

Donde:

$X_{s}^{\prime}=$ cantidad ofrecida con subsidio al productor de carne de cerdo en canal, en toneladas

$\theta_{s}:=$ intercepto de la función de oferta de carne de cerdo en canal $\gamma_{s=\text { pendiente de la función de oferta de carne de }}$ cerdo en canal

$P=$ precio de la carne de cerdo en canal, en pesos por tonelada

$S=$ monto del subsidio al productor de carne de cerdo en canal, en pesos por cada cerdo finalizado y sacrificado en rastro.

Una vez incorporado el subsidio, se generó la función inversa de la oferta:

$$
P_{s i}=P_{s i}\left(X_{s i}\right)=\mathrm{V}_{s i}+\eta_{s i} X s_{d i} ; \beta>0
$$

\section{Donde:}

$P_{s i}=$ precio de oferta de carne de cerdo en canal, en la región $i$, en pesos por tonelada

$\mathrm{X}_{\mathrm{si}}=$ cantidad ofrecida de carne de cerdo en canal, en la región $i$, en toneladas

$V=$ intercepto de la función de oferta de carne de cerdo en canal, en la región $i$

$\eta$ = pendiente de la función de oferta de carne de cerdo en canal, en la región $i$.

La función de cuasi bienestar social para cada región, se definió por el área entre la curva de demanda $\left(Y_{d i}^{*}\right.$ es la cantidad óptima a obtener) y por el área entre la curva de oferta ( es la cantidad óptima a obtener):

$$
\text { [1] } W_{i}\left(Q_{s i}^{*}, Q_{d i}^{*}\right)=\int_{0}^{Y_{d i}^{*}} P_{d i}\left(Y_{d i}\right) d Y_{d i}-\int_{0}^{X_{s i}^{*}} P_{s i}\left(X_{s i}\right) d X_{s i}
$$

$\mathrm{Al}$ incorporar los costos de transporte entre regiones, la función de bienestar social para la n-región, fue:

[2] $\operatorname{Max} \sum_{i=1}^{n}\left[\int_{0}^{Y_{d i}^{*}} P_{d i}\left(Y_{d i}\right) d Y_{d i}-\int_{0}^{X_{s i}^{*}} P_{s i}\left(X_{s i}\right) d X_{s i}\right]-\sum_{i=1}^{n} \sum_{j=1}^{n} X_{i j} T_{i j}$

Donde:

$X i j=$ es la cantidad transportada de la región $i$ a la región $j$, en toneladas de carne de cerdo en canal.

$T_{i j}=$ Costo de transporte de la región $i$ a la región $j$, en pesos por tonelada de carne de cerdo en canal transportada.

Otros componentes del modelo, fueron las restricciones de demanda y las restricciones de oferta. Las primeras, requieren que la suma de la cantidad trans- 
portada a la región $i$ sea mayor o igual que la demanda de dicha región. Esto es:

$$
Y_{8} \leq \sum_{j=1}^{10} X_{i j}, \text {, para toda } i[3]
$$

Las segundas, requirieron que la suma de la cantidad transportada, fuera de la región $i$, sea menor o igual a la producción total de dicha región:

$$
X_{8} \geq \sum_{j=1}^{10} T_{i j} \text {, para toda } i[4]
$$

El modelo (Takaya y Judge 1964:67), supone la existencia de regiones productoras y consumidoras que comercian un bien homogéneo, que para este caso, es la carne de cerdo en canal, mismas que se encuentran separadas, por los costos de transporte, pero no aisladas entre sí.

De forma regional, el resultado de la integral de [1], es decir, el modelo matemático, quedó como sigue:

$\operatorname{Max} V S N=\sum_{i=1}^{8}\left[\lambda_{d} Y_{d}+\frac{1}{2} \omega d Y_{d}^{2}\right]$ Área bajo la curva de demanda

$-\sum_{s=1}^{10}\left[V_{s} X_{s}+\frac{1}{2} \eta_{s} X_{s}^{2}\right]$ Área bajo la curva de oferta

$-\sum_{s=1}^{8}\left[t_{s d} X_{s d}\right]$ Costo de transporte regional-nacional

$-\sum_{s=1}^{2} t_{s d}$ Costo de transporte de importaciones.

\section{OBTENCIÓN DE LOS DATOS}

La información sobre producción y exportaciones de carne en canal de cerdo, se obtuvo por entidad federativa y provino del Servicio de Información Agroalimentaria y Pesquera (SIAP 2016); después, se restó la exportación a la producción de los estados que reportaron y, se sumó la producción de los estados que integran cada una de las regiones para obtener el dato del volumen regional. La información sobre volúmenes importados se obtuvo del SIAP (2016) según claves arancelarias y puntos de acceso al país (aduanas).

Para conocer el consumo (demanda) de cada una de las ocho regiones del país, primero, se obtuvo la población de cada estado del año 2015 del Instituto Nacional de Estadística, Geografía e Informática (INEGI 2015) y del Consejo Nacional de Población (CONAPO 2016). Esta población, se multiplicó la disponibilidad per cápita reportada para la zona por la Confederación de Porcicultores Mexicanos (PORCIMEX)y, fue: Norte 5,8 $\mathrm{kg}$, Centro 17,4 kg, y sur 29,5 kg (PORCIMEX, 2016), la Confederación de Porcicultores sólo divide al país en esas tres regiones; después, se sumó el consumo de cada uno de los estados que integraron cada región.

El precio regional del cerdo en canal, se obtuvo con el precio de cada entidad federativa que integró la región, mismo que se ponderó con la producción (SIAP 2016). El precio del producto, proveniente de EEUU hacia los puntos de internación, se obtuvo del Sistema Nacional de Información e Integración de Mercados (SNIIM) (2016).

El costo de transporte dentro y entre regiones, se calculó al multiplicar la distancia (en kilómetros) por el costo por kilómetro; las distancias se obtuvieron con la herramienta de la Secretaría de Comunicaciones y Trasportes punto a punto (SCT 2016), se consideró cada capital dela entidad federativa como punto de referencia y se ponderaron las distancias en cada región.
El transporte de canales de cerdo es especializado y tuvo un costo por kilómetro de dos dólares (USD) el equivalente a 35 pesos mexicanos en el momento de la investigación (un tipo de cambio de 17,5 pesos mexicanos por dólar estadunidense al 4 de enero de 2016), el cual se obtuvo de forma directa por entrevista a una empresa particular que realiza servicios de transporte de carga especializada.

En las ecuaciones de oferta y demanda regionales de carne de cerdo, se utilizaron elasticidades precio de la demanda y precio de la oferta regionales inelásticas reportadas por Rebollar et al. (2014) y para los puntos de internación se consideraron las elasticidades nacionales reportadas por Pérez (2010). Las funciones precio-cantidad se calcularon como lo indicaron Alston et al. (1995) y Kawaguchi et al. (1997).

Con la utilización de datos sobre carne de cerdo en canal para cada una de las regiones de México, provenientes de fuentes oficiales, primero se obtuvo el modelo base, conocido como modelo óptimo y sus resultados tanto a nivel regional como nacional sobre producción, consumo e importaciones, así también el Valor Social Neto (VSN). Una diferencia porcentual de estimación entre el modelo y los datos observados menor al $10 \%$, ya se considera como aceptable para realizar el análisis de política. Si tal diferencia resulta negativa/positiva, entonces el modelo subestima o sobreestima los resultados. Después se procedió a realizar la estimación del efecto del subsidio sobre dicho mercado.

De acuerdo con PORCIMEX, en 2015 el Gobierno Federal otorgó un subsidio de 5,7 USD (dólares americanos) (100,0 pesos mexicanos)por cada cerdo a sacrificio en rastro, dicho monto se adicionó a la función de oferta en cada una de las regiones, en tanto que la función de demanda quedó sin modificar; cuyos efectos se contrastaron con los del modelo base, centrados en producción, importaciones, consumo, precios óptimos (al productor y al consumidor) y en la magnitud del Valor Social Neto (VSN).

Para el modelo base, el costo de la carne de cerdo importada que se consideró, fue 1,6 USD por kilogramo (27,4 pesos mexicanos), esto es, por kilogramo (obviando proyecciones de incremento en costos de producción internos, producto del aumento en el precio de los insumos por la nueva situación económica de México en 2017: tipo de cambio e incremento en el precio de la gasolina), mismo que se sumó al costo de transporte de los puntos de internación uno y dos.

Toda la información secundaria se procesó y los resultados, tanto del modelo base como del análisis del escenario del subsidio, se obtuvieron con el procedimiento MINOS, contenido en el software del lenguaje de programación GAMS (General Algebriac Modeling System), versión 24.4.2 para Windows 8, Office 2013 y con base en Rosenthal (2008).

\section{RESULTADOS}

El modelo óptimo o modelo base (Tabla II), subestimó a la producción nacional y regional de carne de cerdo en canal en $0,4 \%$, esto es, que el volumen nacional 
de producción óptima fue menor al observado en 4655 t, significa que los resultados del modelo óptimo fueron suficientemente cercanos a lo observado en ese año (2015).

El modelo sobreestimó al consumo nacional y regional de esta carne en $0,8 \%$, al pasar de 2100,5 miles de $t$ como lo observado en 2015 a 2117,7 miles de t dadas por el modelo base. El modelo sobreestimó a la variable importaciones de carne en canal en 2,5\%, pues el dato observado, en el año de análisis, fue menor que el resultado dado por el modelo equivalente a 2189,1 miles de t. Sin existencia de distorsiones en el mercado y distribución del producto y bajo condiciones óptimas, el modelo base indica una reducción regional en la producción de carne de cerdo en canal, en las regiones Centro-oeste (CO), Centro-este (CE) y la Noroeste (NO), equivalente a 339, 1020 y 186 t, en favor de mayor bienestar social (VSN).

Con el subsidio a la porcicultura mexicana (Tabla II), la producción tanto a nivel región como nacional de carne de cerdo en canal, se incrementó. La producción nacional, con referencia al modelo base, aumentó en

Tabla II. Efectos del subsidio sobre producción, consumo e importaciones de carne de cerdo en canal, en México (Effects of subsidy on pork production, consumption and imports, in Mexico)

\begin{tabular}{|c|c|c|}
\hline Región & Modelo base & Con subsidio \\
\hline & \multicolumn{2}{|c|}{ Producción (t) } \\
\hline Noroeste (NO) & 193553 & 193554 \\
\hline Norte (NR) & 33145 & 33146 \\
\hline Noreste (NE) & 31426 & 31428 \\
\hline Centro-occidente (CO) & 419409 & 419411 \\
\hline Centro-este (CE) & 232257 & 232271 \\
\hline Sur (SU) & 76192 & 76193 \\
\hline Oriente (OR) & 128866 & 128919 \\
\hline Península de Yucatán (PE) & 105539 & 105546 \\
\hline \multirow[t]{2}{*}{ Nacional } & 1220387 & 1220468 \\
\hline & \multicolumn{2}{|c|}{ Importaciones (t) } \\
\hline Punto de internación 1 & 810588 & 810801 \\
\hline Punto de internación 2 & 86777 & 86800 \\
\hline \multirow[t]{2}{*}{ Nacional } & 897365 & 897601 \\
\hline & \multicolumn{2}{|c|}{ Consumo (t) () } \\
\hline Noroeste (NO) & 80423 & 80424 \\
\hline Norte (NR) & 126296 & 126312 \\
\hline Noreste (NE) & 50538 & 54544 \\
\hline Centro-occidente (CO) & 363390 & 363460 \\
\hline Centro - este & 774512 & 774637 \\
\hline Sur (SU) & 284747 & 284772 \\
\hline Oriente (OR) & 303891 & 303940 \\
\hline Península de Yucatán (PE) & 133975 & 133979 \\
\hline Nacional & 2117772 & 2122068 \\
\hline VSN (MDD) & 216563 & 216575 \\
\hline
\end{tabular}

Fuente: resultados de la salida del GAMS, con datos de 2015. MDD: millones de dólares.
$0,07 \%(81 \mathrm{t})$ al pasar de 1220,4 miles de $t$, sin subsidio a 1 220,5 miles de $t$ con subsidio; el volumen de compras externas de esta carne, tanto totales como las que entran por los dos puntos de internación, se incrementó en $0,03 \%(236 \mathrm{t})$ y el consumo total nacional lo hizo en $0,006 \%$ (776 t). Con el subsidio a la producción, el VSN se incrementó en 0,006\% (12 MMD).

Durante el periodo de estudio, el subsidio a la producción de carne de cerdo en canal, también generó variaciones sobre el nivel de precios óptimos tanto al productor como el consumidor de dicho producto (Tabla IV).

Con relación al modelo base o modelo óptimo, el subsidio redujo tanto el precio que recibió el productor como el que pagó el consumidor por este producto pecuario en todas las regiones productoras (se incluye los puntos de internación de las importaciones) y consumidoras de México. Por región, el diferencial de precios (al consumidor y al productor), por el subsidio, fue distinto. Para la región NO 12,5\% (198,9 USD/t

Tabla III. Producción, consumo e importaciones de carne de cerdo, en México, bajo condiciones óptimas (Production, consumption an imports of pork in Mexico, under optimum conditions)

\begin{tabular}{|c|c|c|}
\hline Región & Datos observados & Modelo base \\
\hline & Producción (t) & \\
\hline Noroeste (NO) & 193739 & 193553 \\
\hline Norte (NR) & 33393 & 33145 \\
\hline Noreste (NE) & 31633 & 31426 \\
\hline Centro-occidente (CO) & 419748 & 419409 \\
\hline Centro - este (CE) & 233277 & 232257 \\
\hline Sur (SU) & 76240 & 76192 \\
\hline Oriente (OR) & 130667 & 128866 \\
\hline Península de Yucatán (PE) & 106345 & 105539 \\
\hline \multirow[t]{2}{*}{ Nacional } & 1225042 & 1220387 \\
\hline & Importaciones (t) & \\
\hline Punto de internación 1 & 788977 & 810588 \\
\hline Punto de internación 2 & 86497 & 86777 \\
\hline \multirow[t]{2}{*}{ Nacional } & 875474 & 897365 \\
\hline & Consumo (t) & \\
\hline Noroeste (NO) & 80397 & 80423 \\
\hline Norte (NR) & 124481 & 126296 \\
\hline Noreste (NE) & 50139 & 50538 \\
\hline Centro-occidente (CO) & 354833 & 363390 \\
\hline Centro - este & 76221 & 774512 \\
\hline Sur (SU) & 284737 & 284747 \\
\hline Oriente (OR) & 303029 & 303891 \\
\hline Península de Yucatán (PE) & 133680 & 133975 \\
\hline Nacional & 2100517 & 2117772 \\
\hline VSN (MDD) & 215787 & 216563 \\
\hline
\end{tabular}

Fuente: resultados de la salida del GAMS, con datos de 2015. MDD: millones de dólares. 
Tabla IV. Efectos del subsidio sobre precios al productor y al consumidor de carne de cerdo en México, cifras en USD/t (Subsidy effects on consumer and producer prices of pork in Mexico, USD/t data).

\begin{tabular}{lccc}
\hline & \multicolumn{2}{c}{ Modelo base } & \\
\hline Región & Productor & Consumidor & Productor \\
Noroeste (NO) & 1596,0 & 1794,9 & 1592,5 \\
Norte (NR) & 1839.6 & 1925,7 & 1836,1 \\
Noreste (NE) & 1853,5 & 1862,9 & 1849,9 \\
Centro-occidente (CO) & 1911,1 & 1954,3 & 1907,6 \\
Centro-este (CE) & 1983,3 & 2010,9 & 1969,4 \\
Sur (SU) & 1994,4 & 2093,7 & 1959,4 \\
Oriente (OR) & 2034,8 & 2090,9 & 1950,9 \\
Península (PE) & 2154,2 & 2190,9 & 2031,3 \\
Punto de internación 1 (PI1) & 1781,1 & & 2150,7 \\
Punto de internación 2(PI2) & 1595,7 & & 2090,9 \\
\hline
\end{tabular}

Fuente: elaboración propia, con base en la salida de resultados del modelo base y modelo con subsidio.

como la diferencia entre 1794,9 y 1596,0$)$, en la NR $4,7 \%$, para la PE $1,7 \%$ y así sucesivamente.

Con subsidio a la producción (oferta) de carne de cerdo en canal, en la región NO de México, el productor habría dejado de percibir 3,5 USD/t (-0,2\%), el de la CE 13,9 USD/t y para el resto de las regiones el resultado fue similar; pero el efecto del subsidio sobre el consumidor fue diferente; con subsidio, el consumidor de la región NO dejó de pagar 3,4 USD/t de carne de cerdo en canal con relación al modelo base, el de la región CE 4,0 USD/t y en forma similar para el resto de las regiones; por lo que el consumidor habría sido el más beneficiado al dejar de pagar menos USD por unidad de producto, mientras que el productor habría recibido un diferencial de precio menor en la venta, con relación a lo que ahorró el consumidor.

\section{DISCUSIÓN}

Los resultados del modelo base o modelo óptimo, maximizaron el Valor Social Neto, con relación al nivel observado en el año de análisis; el VSN del modelo óptimo, fue 0,006\% superior al observado en el año de análisis, equivalente a $12 \mathrm{MDD}$ una vez considerado el tipo de cambio de ese periodo. Dicha optimización sugirió reducir producción nacional de carne de cerdo en canal en 0,4\% (4 $655 \mathrm{t}$ ), que habría de sustituirse con importaciones. $\mathrm{Al}$ respecto, tal volumen de compras externas representó 21,9 miles de $t(2,5 \%)$ adicionales a las observadas en 2015, entre las que se encuentra inmersa la reducción de la producción.

La disminución del volumen nacional de carne de cerdo en canal, se redistribuyó en cada una de las regiones productoras, así, de las 4655 t de carne que habría dejar de producir, la región Oriente $(\mathrm{OR})$ de México participaría con 1801 t, la Centro-este (CE) en 1 020, la Península de Yucatán (PE) con 806, la Centrooccidente (CO) en 339 t y sucesivamente con el resto de las regiones pero en cantidades distintas.

De acuerdo con la Tabla III, la maximización del VSN condujo al aumento del consumo total nacional de este subproducto pecuario en $4296 \mathrm{t}(0,2 \%)$. Esta variación se observó en regiones con mayor dinamismo como la CO, CE y OR tal y como lo afirmaron Rebollar Rebollar et al. (2014) en un estudio sobre la porcicultura mexicana.

\section{ANÁLISIS DEL EFECTO DEL SUBSIDIO}

En este trabajo y para el año de análisis, el subsidio no se vinculó bajo alguna figura de protección en México, debido a que no está establecido legalmente como apoyo permanente, contrario a lo que afirmaron Candek et al. (2018). En lo general, el subsidio es fuente de desplazamiento de la curva de oferta de cualquier producto en un mercado; un subsidio desplaza hacia la derecha la curva de oferta del producto en cuestión (Graue 2006; Parkin y Loría 2015). En este estudio, el valor del subsidio se insertó directamente en el modelo de oferta, para generar después, el sistema de oferta inverso. Como ya se mencionó, no se adicionó a los costos de transporte; por lo que los resultados, indudablemente, son distintos con relación a que si dicho subsidio se analiza en la función de oferta a que si se aplica a costos de transporte, como lo hicieron Velázquez et al. (2016).

Cuando un subsidio se adiciona en la función de oferta del producto, sin considerar el análisis completo del modelo, según Graue (2006), la curva de oferta del producto se desplaza hacia la derecha y la producción aumenta con las respectivas consecuencias en los precios tanto al productor como al consumidor; sin embargo, bajo las condiciones planteadas en esta investigación, se considera que el efecto del subsidio es más efectivo cuando se inserta en la función de oferta, porque se vislumbra como una situación de largo plazo, debido a que el desplazamiento de la curva de la oferta no es inmediato a la aplicación del subsidio sino que se realiza hasta la obtención del producto terminado.

Con base en la información de la Tabla III, el subsidio incrementaría la producción, importaciones y consumo nacional de carne de cerdo en canal en 0,007, 0,03 y $0,006 \%$ con relación al modelo base; que en términos absolutos significa aumentos de 81, 236 y $4296 \mathrm{t}$; 
el efecto del subsidio no sería significativo en el mercado, pero sí tendría diferencias ligeras, presenciándose poco más tanto en consumo tanto nacional como en cada una de las regiones. Con el subsidio, el bienestar de la sociedad $t$ (VSN) también aumentaría en 0,006\% (12 MDD), con relación al modelo óptimo. Al respecto, Velázquez et al. (2016), utilizaron una metodología similar en la que adicionaron el subsidio (USD 5,7) a costos de transporte y concluyeron que tanto producción, consumo y VSN se incrementarían con relación al modelo óptimo, pero el volumen de importaciones disminuiría. En ese estudio, el VSN obtenido fue mayor al de este trabajo.

Con relación a los precios, de acuerdo con la Tabla IV, sin subsidio y con subsidio, las regiones Oriente (OR) y Península de Yucatán (PE) presentarían los mayores precios al productor; en tanto que las regiones Noroeste (NO) y Noreste (NE) se beneficiarían al pagar los precios más bajos al consumidor de carne de cerdo en canal, debido a los costos de transporte y al precio del producto importado.

En adición, bajo las condiciones planteadas, con subsidio el precio que pagaría el consumidor por cada tonelada de carne de cerdo en canal sería menor con relación al del modelo base; en otras palabras, con subsidio, el consumidor de este producto se beneficiaría al pagar un precio menor por cada unidad de producto con referencia a la variable de análisis que fue el modelo base. Por ejemplo, sin subsidio a la producción de carne de cerdo en canal, en 2015, el consumidor de la región de la PE (Península de Yucatán) pagó 2190,9 USD/t, con subsidio desembolsaría2 187,4 USD/t; es decir, una reducción en el precio de $0,2 \%$, equivalente a 3,5 USD/t, el de la región NO 10,2 USD/t y así con el resto de las regiones. Si bien, el efecto de la política aumentaría el consumo, también se espera que el crecimiento de la población y cambios en gustos y preferencias de los consumidores, mantengan el dinamismo en tal variable (Rebollar et al. 2014; Sosa et al. 2017).

Por su parte, el subsidio también tendría efectos en el precio que recibirían los productores de carne de cerdo en canal en México en 2015. Al adicionar el valor del subsidio a la función de oferta dentro del modelo, se tuvo un efecto de menor precio por unidad de volumen que recibiría el productor en cada una de las regiones.

De acuerdo con la teoría del mercado, a mayor producción, el productor recibe un menor precio; así, el productor de carne de cerdo en canal de la región Noroeste de México, con subsidio recibiría 3,5 USD/t menos que sin subsidio, lo mismo para el resto de las regiones; sin embargo, debido a que el dinamismo tanto en producción como en consumo de este subproducto pecuario es diferente en todo el territorio nacional (Rebollar et al. 2014), es posible afirmar que los consumidores serían los beneficiados por la política, al pagar un precio menor por esta carne en términos relativos. En otras palabras, el aumento en el consumo y la reducción de los precios de esta carne sería mayor con relación a la producción tanto nacional como a nivel región, debido a la distinta dinámica interregional de esta actividad, como lo afirmaron García et al. (2005); Bobadilla et al. (2010) y Rebollar et al. (2015).

Si bien, aunque el productor de carne de cerdo en canal recibiría un precio menor por el producto con subsidio, el efecto total sería que, con demandas y ofertas inelásticas, tanto el volumen nacional producido de esta carne y el consumo aumentarían, los precios disminuirían y el bienestar de la sociedad incrementaría en menos de una unidad porcentual; lo anterior constituye una condición necesaria como política viable de continuidad, de largo plazo, por parte del gobierno de México siempre y cuando hubiese suficiente presupuesto federal para ello y que el gobierno acertara en aprobar tal política.

\section{CONCLUSIÓN}

Ante demandas y ofertas inelásticas, la aplicación de un subsidio a la producción mexicana de carne de cerdo en canal, incrementaría la producción, importaciones y consumo tanto nacional como en cada una de las regiones. Con base en los resultados que se obtuvieron, con el subsidio, el bienestar de la sociedad, medida por el Valor Social Neto se incrementaría con relación al modelo óptimo, por tanto, el subsidio genera beneficios.

Cuando el subsidio se adiciona a la función de oferta del producto, el modelo indica que los productores recibirían menores precios por unidad de volumen y los consumidores pagarían un precio inferior que sin subsidio, con tales resultados y ante la inelasticidad de la demanda y la oferta de carne de cerdo en canal, los consumidores y la sociedad en general, se verían beneficiados. El mercado no es tan sensible a la aplicación del subsidio, pero la política es viable y se recomienda su continuidad.

\section{AGRADECIMIENTOS}

Los resultados de esta contribución, son parte del proyecto de investigación aprobado por la Universidad Autónoma del Estado de México, clave UAEM 4233/2016SF.

\section{BIBLIOGRAFÍA}

Alston, JM, Norton, GW \& Prdey, PG 1995, Science Under Scarcity: Principles and Practice for Agricultural Research Evaluation and Priority Setting, Cornell University Press, Ithaca, New York, USA.

Bassols, AB 1999, El Desarrollo Regional de México: teoría y práctica Libros de la Revista Problemas del Desarrollo, Instituto de Investigaciones Económicas, UNAM, México.

Bobadilla, SEE, Espinoza, OA, y Martínez, CFE 2010, "Dinámica de la producción porcina en México de 1980 a 2008, Revista Mexicana de Ciencias Pecuarias, vol1, no. 3, pp. 251-268.

Consejo Nacional de Población (CONAPO) 2016, Consultado 22 oct 2016, http://www.conapo.gob.mx/.

Fideicomisos Instituidos en Relación a la Agricultura (FIRA) 2017. Consultado 29 jun 2017, https://www.gob.mx/cms/uploads/attachment/ file/200634/Panorama_Agroalimentario_Carne_de_Cerdo_2016. pdf.

García, SJA, Rebollar, RS, y Rodríguez, LG 2005, "Integración vertical y competitividad del sector porcino en México", Comercio exterior, vol5, no. 55, pp. 524-532. 
Grave, RAL, 2006, Microeconomía. Enfoque de negocios, 1 ra ed, México, D. F.

Instituto Nacional de Estadística Geografía e Informática (INEGI) 2015, Censo de Población y Vivienda 2010: 2015, Consultado 15 feb 2015, http://www.inegi.org.mx/.

Kawaguchi, T, Susuki, N, \& Kaiser, M 1997, "A Spatial equilibrium model for imperfectly competitive milk markets". Am J Agr Econ, vol79, pp.851-859.

Čandek, P. M., Giusto, A. C., Cosola, C. and Fontanesi, L 2018, "Improving sustainability of local pig breeds using quality labels - case review and trademark development in project TREASURE". Archivos de Zootecnia. PROCEEDINGS IX Simposio Internacional sobre el Cerdo Mediterráneo: 235-238. 2018

Martínez, DMA, y Hernández OJ 2012, "Importaciones de granos básicos y precio interno en México: un enfoque de sistema de demanda inverso", Agricultura, Sociedad y Desarrollo, vol 9, pp. 401-410.

Parkin, M, y Loría, E 2015, Microeconomía. Versión para Latinoamérica, 1 lava ed, Pearson, Ciudad de México, México.

Pérez, VFC, García, MR, Martínez, DMA, Mora, FJS, Vaquera, HH, y González, EA 2010, "Efecto de las importaciones de la carne de porcino en el mercado mexicano, 1961-2007", Revista Mexicana de Ciencias Pecuarias, vol 1, no. 2, pp.115-126.

Porcicultores Mexicanos (PORCIMEX) 2017, Estadísticas: 2017, Consultado 29 Jun 2017, http://www.porcimex.org/estadisticas/ nprodmundial.htm.

Rebollar, RA, Gómez, TG, Hernández, MJ, Rebollar, RS, y González, RFJ 2014, "Comportamiento de la oferta y demanda regional de carne de cerdo en canal en México, 1994-2012", Revista Mexicana de Ciencias Pecuarias, vol5, no. 4, pp. 377-392.

Rebollar, RA, Gómez, TG, Rebollar, RS, Hernández, MJ, y González, RFJ 2015, "Dinámica regional de la producción porcina en México, 1994-2012", Agrociencia, vol 49, pp. 455-473.
Rosenthal, ER 2008, GAMS, A User's Guide, GAMS Development Corporation, Washington, D. C., USA.

Secretaría de Comunicaciones y Transportes (S.C.T) 2016, Rutas punto a punto: 2016, Consultado 20 Ago 2016, http://www.sct.gob.mx/ rutaspuntoapunto.

Servicio de Información Agroalimentaria y Pesquera (SIAP) 2016, Estadísticas: 2016, Consultado 16 Feb 2016, http://www.siap.gob. $\mathrm{mx} /$ optestadisticasiacon2016parcialsiacon-zip/.

Servicio Nacional de Sanidad, Inocuidad y Calidad Agroalimentaria (SENASICA), 2015, Censo Pecuario 2014: 2015, Consultado 6 ene 2015, http://www.senasica.gob.mx/.

Sistema Nacional de Información e Integración de Mercados-Secretaria de Economía (SNIIM-SE) 2016, Consultado 21 ene 2016, http:// www.economia-sniim.gob.mx/nuevo/mapa.asp.

Sosa, UME, Martínez, CFE, Espinosa, GJA, y Buendía, RG 2017, "Contribución del sector pecuario a la economía mexicana. Un análisis desde la Matriz Insumo Producto, Revista Mexicana de Ciencias Pecuarias, vol8, no. 1, pp. 31-41.

Takayama, T, \&Judge, GG 1964, "Spatial equilibrium and quadratic programming", Journal of Farm Economics, vol46, no. 1, pp. 67-93.

Velázquez, VHH, Gómez, TG, Rebollar, RS, y Martínez, CFE 2016. "Los subsidios y su efecto en la porcicultura mexicana", Revista Mexicana de Ciencias Pecuarias, En revisión. 